\title{
Los más pequeños toman la palabra: la Escuela Infantil que a ellos les gustaría
}

\author{
The little ones take the word: \\ the pre-school that they would like \\ Ana CASTRO ZUBIZARRETA y Nuria del Mar MANZANARES CEBALLOS \\ Universidad de Cantabria
}

Recibido: Noviembre 2014

Evaluado: Enero 2015

Aceptado: Enero 2015

\section{Resumen}

El derecho a ser escuchado y participar en aquellos asuntos que afectan a los niños es un derecho fundamental de la infancia escasamente respetado por el adulto, quien tiende a silenciar su voz, infravalorando su visión y posible aportación. Ante esta realidad, la Escuela Infantil se erige como un marco privilegiado para la escucha y la participación de la infancia en aquellos temas que les afectan, contribuyendo a la formación de ciudadanos democráticos. A partir de un marco de escucha donde se concibe al pequeño como un sujeto activo, competente y experto en su propia vida, se ha llevado a cabo una investigación que ha adoptado el formato de estudio de caso, en la que se ha recogido a través de las técnicas del dibujo y de las conversaciones con niños, la visión que poseen los más pequeños sobre la Escuela Infantil, analizando el bienestar del niño en el contexto escolar, el sentido y finalidad que los niños otorgan a la escuela; el grado de satisfacción con la misma; sus gustos y preferencias en el contexto escolar; la autonomía y libertad que dicen poseer en la escuela; su visión sobre los aspectos organizativos que rigen el funcionamiento del centro y su perspectiva sobre cómo es y cómo les gustaría que fuera la participación de las familias en la escuela. Finalmente, se recogen sus propuestas de mejora para hacer de la Escuela Infantil a la que asisten su escuela ideal. Los resultados del estudio ponen de manifiesto la demanda de los niños de una Escuela Infantil diferente a la que conocen y la existencia de un pensamiento divergente del pensamiento adulto que es necesario escuchar y tener en consideración, siendo sus aportaciones de gran valía para poder iniciar procesos de innovación y mejora educativa.

Palabas clave: perspectiva infantil, educación Infantil, participación infantil, innovación educativa.
Abstract
The right to be listened and participate in all those matters which affect children is a fundamental right scarcely respected by adults, who tend to silence them, and underrate their views and their possible contribution. Dealing with this reality, Pre-school stands as a privileged setting for listening to the children and allowing them to participate in those issues that affect them, contributing to the formation of future democratic citizens. From a framework 
of listening, where children are conceived as active and competent subjects, as experts in their own lives, we have conducted an investigation which has adopted the format a case study. Through drawing techniques and discussions with children we have gathered their views about the Pre-school, analysing children's well-being in the school context; the meaning and purpose they give to school; the level of satisfaction with it; their likes and preferences in the school context; the autonomy and freedom they say they have at school; their vision about organizational aspects that rule the school and their perspective about the real and their desired involvement of families. Finally, we gather their proposals for improvement in order to make their pre-school and ideal pre-school. The results of this study show that children are demanding a different kind of pre-school from the one they know, and the existence of a divergent thought from that of the adult, which needs to be listened to and taken into consideration, being their proposals valuable to start innovation processes and educational improvement.

Keywords: children's views, pre-school education, child participation, educative innovation.

En los últimos años se ha producido un incremento significativo del interés por recoger la visión del niño en aquellos temas que afectan a su vida, presentando a la infancia como un colectivo competente, activo, con derechos, con capacidad de tomar decisiones y por lo tanto, de participar como ciudadanos de pleno derecho en la sociedad en la que viven (Bruner, 1996; Dahlberg, Moss y Pence, 1999; Pascal y Bertram, 2009; Harcourt y Einarsdottir, 2011). Tal y como señalan Clark, McQuail y Moss (2003) este cambio de pensamiento en torno a la concepción de la infancia, vinculado al enfoque de la escucha a la infancia o la participación infantil, ha tenido repercusiones importantes en el ámbito legal, político, económico y académico.

Concretamente, en el ámbito legal y político, la Convención de los Derechos del Niño (1989), la primera ley internacional sobre los derechos de la Infancia, sienta las bases del derecho de los pequeños a opinar, a ser tenidos en cuenta en aquellos temas que les afectan, a ser respetados y escuchados, tal y como se refleja en el artículo 12 de la citada Convención. Sin embargo, el desarrollo efectivo de este derecho, queda limitado y difuminado en una práctica diaria donde la voz infantil sigue siendo silenciada e infravalorada bajo el poder del adulto (Argos, Ezquerra y Castro, 2011) ya que, como bien señalan Liebel, y Saadi, (2012), tener derechos no supone poder practicarlos.

Un ámbito donde paradójicamente ha tenido repercusión este cambio conceptual de acercamiento a la infancia es el económico, donde se apunta a la infancia como un colectivo consumidor, diferenciado del adulto al que hay que escuchar para identificar sus intereses, gustos y preferencias (Clark, McQuail y Moss, 2003). Evidentemente, es necesario replantear los fines del proceso de escucha, erigiéndose el ámbito académico y más concretamente el escolar, como un contexto privilegiado para poder promover procesos de escucha que favorezcan el desarrollo de ciudadanos democráticos y participativos. El contexto escolar se presenta por lo tanto, determinante para el aprendizaje de los derechos y los deberes que competen al desarrollo individual y colectivo. 
Será, en este contexto, donde se enmarque la presente investigación, que nace con la pretensión de recoger la perspectiva que poseen los niños sobre la Escuela Infantil a la que asisten, escuchando las aportaciones de los principales protagonistas de la escuela: los pequeños.

\section{Procesos de escucha a la infancia en el contexto escolar}

Referirse a los niños como actores sociales, implica la no discriminación y su participación real y efectiva en la sociedad de su tiempo (Rubiano, 2010). El horizonte hacia el cual transitar marcado en la Convención de los Derechos del Niño (1989), no está exento de dificultades y desafíos que surgen en la implementación de un derecho básico como es el de ser escuchado y que ha promovido la elaboración de las denominadas Observaciones generales del Comité de los Derechos del Niño, donde se explicitan preocupaciones y recomendaciones para que los derechos recogidos en la citada Convención, puedan ser ejercidos. En este sentido, son de especial interés las Observaciones generales $\mathrm{n}^{\circ} 7$ del Comité de los Derechos del Niño (2005), donde se incide en el respeto a las opiniones e intereses de los niños pequeños, insistiendo en que los niños de más corta edad pueden hacer elecciones y comunicar sus sentimientos, ideas y deseos de múltiples formas, mucho antes de que puedan comunicarse mediante las convenciones del lenguaje hablado o escrito, así como las Observaciones generales $\mathrm{n}^{\circ} 12$ (2009), donde se plantea que uno de los mayores obstáculos para hacer efectivo el derecho del niño a ser escuchado es la actitud del adulto que sigue infravalorando las opiniones de los pequeños.

Tal y como señalan Alderson (2000) y Clark, McQuail y Moss (2003), la escucha puede limitarse a un proceso pasivo. Sin embargo, a nuestro entender, los procesos de escucha debieran encaminarse hacia la consecución de procesos activos de intercambio de significados (Rinaldi, 2001; Clark y Moss, 2001) que partan de la necesidad comprender y asumir la exigencia ética del reconocimiento del otro, otorgando a los niños la palabra, ayudando a expresarla en sus múltiples formas de presentación.

Esta definición es la que acerca este concepto de escucha al de participación, entendiéndola como el proceso de intercambiar pensamientos e ideas y compartir las decisiones que afectan la vida propia y la vida de la comunidad en la cual se vive, cuyos tipos y niveles han sido ampliamente abordados por Hart (1993) y Trilla y Novella (2011) y que implica, inevitablemente en primer lugar, constatar que se forma parte de algo (Novella, 2012), y en segundo lugar, lograr que se produzca un reparto de poder (Miller, 1997) en la línea de lo ya apuntado por Christensen y Prout (2002) cuando acuñaron el término de la simetría ética y que pone de relieve la necesidad de mitigar el poder del adulto sobre el niño (Argos, Ezquerra y Castro, 2011). Asimismo, estas premisas no son efectivas si no se consigue transmitir a los niños confianza en sí mismos fomentando su iniciativa y el ejercicio de un rol activo, donde se les posibilite espacios y marcos de escucha para poder demostrar su capacidad de expresar opiniones y de tomar decisiones en los asuntos que les competen.

Desde el contexto escolar, han sido diversas, las aportaciones que se han acercado a los procesos de escucha y participación infantil, sustentadas en la necesidad de 
repensar el lugar que ocupa el alumnado en la escuela, tal y como señala Bertolini (2011), recogiendo la diversidad de voces existentes en el contexto escolar (Fielding, 2011). Dar voz a los niños en la escuela supone mejorar la vida democrática y pedagógica de las aulas, formar ciudadanos críticos y participativos (Rudduck y Flutter,2007) quienes durante el ejercicio de su derecho a ser escuchados, aprenden a dialogar, a intercambiar puntos de vista, a respetar normas y consensuar acuerdos (Apud, 2007). Supone por tanto, concebir al alumnado como un colectivo esencial para promover iniciativas de cambio y mejora escolar implicándolo en el centro educativo, incrementándose así su sentido de pertenencia y responsabilidad con el mismo.

Diversas son las prácticas en el ámbito escolar o académico que intentan romper con lo que Fine (1991) denominó el silenciamiento de los estudiantes en las escuelas. Sin embargo, las investigaciones que han centrado su atención en recoger la opinión del alumnado han tenido un impacto práctico y real limitado (Messiou, 2013; Mitra, 2003; Rudduck, y Flutter, 2000) encontrando dentro del colectivo estudiantil que la voz de los más pequeños es la más marginada. Consideramos que para poder avanzar y mejorar esta realidad, es preciso transmitir nuevas actitudes en la población adulta que reviertan en un ejercicio efectivo en la vida cotidiana de los niños de sus derechos y que infunda en el pensamiento del adulto que ofrecer procesos de escucha y participación a la infancia permitirá el desarrollo de ciudadanos responsables, democráticos y participativos en la sociedad en la que les ha tocado vivir.

Al respecto, es importante señalar que los procesos de escucha y participación son mayoritarios en etapas educativas superiores, siendo en la Educación Secundaria y sobre todo, en el contexto universitario, donde la opinión del alumnado goza de mayores cotas de influencia y una mayor tradición (Caballero et at; 1999; Saez, 2000; Ruiz de Miguel yOliveros, 2006; Molero, 2007; Coterón, Franco y Gil, 2012; Jiménez y Navaridas, 2012).

En la última década, se han incrementado la investigaciones que centran su atención en recoger la opinión del alumnado escolarizado en la Educación Primaria (Parrilla y De la Fuente, 2013; Doval, Martínez-Figueira y Raposo, 2013) siendo muy limitada la aparición de estudios que recogen la mirada de los más pequeños: el alumnado de la etapa de Educación Infantil (Clark, y Moss, 2001; Argos, Ezquerra y Castro, 2011; Castro, Ezquerra y Argos, 2012a; Castro, Ezquerra y Argos, 2012b y Guzmán, 2013).

A pesar de la escasez de estudios que recogen la opinión de los más pequeños, consideramos importante rescatar su visión sobre aquellos temas que experimentan en primera persona y de los cuales son protagonistas y por tanto también, expertos. Por ello, nos planteamos la siguiente investigación, presentándose a continuación los objetivos que la orientan:

- Conocer la percepción y visión que tienen los niños sobre la Escuela Infantil.

- Promover el derecho de los más pequeños a participar, a ser escuchados y ser tenidos en cuenta. 
- Proporcionar propuestas de mejora sustentadas en las aportaciones de los pequeños que incidan en los procesos de innovación y mejora de la práctica docente.

\section{Método}

\section{Ubicación paradigmática}

La investigación se inscribe bajo el paradigma de la investigación cualitativa mediante la metodología del estudio de caso.

\section{Muestra}

La muestra estuvo conformada por dos unidades del último curso ( 5 años) de la etapa de Educación Infantil de un colegio público de Cantabria a las que se tuvo acceso una vez solicitado el consentimiento informado del profesorado, familias y los propios niños, que participaron de forma voluntaria durante todo el proceso de la investigación. Treinta pequeños fueron los que finalmente participaron en la investigación.

A pesar de que la muestra sea limitada, consideramos que los resultados obtenidos pueden ser representativos y de interés para la comunidad educativa ya que se presenta un proceso de escucha a la infancia que pretende abrir, a través de los resultados que ofrece, la reflexión sobre cómo mejorar la Escuela Infantil.

\section{Técnicas de recogida de la información}

Los instrumentos seleccionados para recoger la perspectiva infantil fueron las conversaciones con niños y la técnica del dibujo. Técnicas de recogida de datos utilizadas en otras investigaciones (Borzone y Rosemberg, 2000; Young y Barrett, 2001; Dockett y Perry, 2005; Castro, Ezquerra y Argos, 2012a) y que permiten recoger la opinión de los más pequeños con eficacia superando la excesiva dependencia de las habilidades verbales de otras técnicas de recogida de información.

a) Las conversaciones con niños: Los pequeños hablaron con una de las investigadoras por parejas con el objeto de facilitar su comodidad y mitigar, en la medida de lo posible, la disparidad del poder adulto frente a los niños. Las conversaciones giraron en torno a los siguientes ejes temáticos: Bienestar del niño en el contexto escolar; sentido y finalidad de la escuela; grado de satisfacción con la escuela; gustos y preferencias en el contexto escolar; autonomía y libertad otorgadas a los niños en la escuela; aspectos organizativos; participación de las familias en el contexto escolar y formulación de propuestas de mejora.

b) Dibujo: La actividad de dibujo planteada consistió en entregar a los niños un folio en blanco y pedirles que dibujasen cómo sería para ellos su escuela ideal, explicándoles que en el dibujo tenían que pintar todo aquello que les 
gustaría que tuviese su escuela sirviendo esta técnica como complemento a los tópicos planteados a través de las conversaciones.

\section{Procedimiento}

Los pasos que guiaron la investigación tuvieron muy presentes en todo momento las cuestiones éticas que deben orientar cualquier investigación con niños, velando en todo momento por su seguridad, libertad y capacidad de decisión en torno a la participación en la investigación durante todas las fases que presentamos a continuación:

- La obtención del consentimiento informado de los referentes adultos del niño: en primer lugar, nos dirigimos tanto a la dirección del colegio como a las tutoras de las aulas de cinco años para explicarles el propósito de la investigación. Después de obtener su permiso para desarrollar el estudio en el centro, las tutoras de cada aula mandaron una circular a los padres donde se les explicaba el motivo y las actividades de la investigación, solicitando su consentimiento y aprobación.

- La obtención del consentimiento informado de los niños: Conseguir el consentimiento por parte de los adultos de la participación de los pequeños en el estudio no posibilita, por sí mismo, desde el enfoque en el que nos apoyamos, la participación del niño en la investigación. Es necesario, lograr que los pequeños comprendan el propósito de la investigación en la que se les propone ser una pieza importante de la misma y donde su consentimiento a participar libremente, determinará el devenir del estudio. Para ello, se fijó un día junto a las maestras para informar a los niños y constatar también su voluntad de participar. En este día una de las investigadoras, se presentó ante los alumnos durante las asambleas de los dos grupos del aula de 5 años explicándoles el motivo de su presencia ese día en su aula y la razón por la que quería que participaran, insistiendo en todo momento en que era algo voluntario e indicándoles que las tareas implicadas eran contestar a unas preguntas por parejas y realizar un dibujo.

- La recogida de datos para iniciar el proceso de escucha: Con el objeto de mantener las conversaciones con niños en un entorno cómodo y conocido para ellos se optó por llevar a cabo las conversaciones en un rincón del aula que entendíamos como seguro y familiar y que dio pie a un intercambio dialógico rico en significado. Todas las conversaciones fueron grabadas en audio, de lo cual también fueron informados todos los agentes implicados, y tuvieron una duración aproximada de entre diez y quince minutos. La actividad de dibujo se realizó en el grupo-clase de forma individual contando con el apoyo de las maestras tutoras sin la presencia directa del equipo investigador que se mantuvo en un segundo plano para no influir en sus producciones.

- De las aportaciones individuales de los niños a la emisión un mensaje compartido: Consideramos importante que los niños fueran partícipes de todo el proceso de investigación, recogiendo no sólo sus aportaciones sino devolviendo 
a través de un mural en una asamblea de aula, el análisis del contenido recogido, consensuando el resultado final de sus aportaciones. Dicho mural fue entregado a la Dirección del Centro por parte de los niños con el propósito de que su mensaje fuera tenido en cuenta y pudiera dar origen a futuros cambios y procesos de mejora en el colegio.

\section{El análisis de la información}

El tratamiento de la información recogida fue analizada a través del programa Atlas.ti. En primera instancia, se llevó a cabo la segmentación del cuerpo de datos (tanto conversaciones como dibujos), para posteriormente ser codificado en unidades de significado de interés para los propósitos de esta investigación, seleccionando y describiendo con posterioridad, aquellos considerados más representativos.

Las categorías de análisis obtenidas sobre el tema de estudio abordado fueron las siguientes:

- Bienestar del niño en el contexto escolar.

- Estado emocional que acompaña la asistencia del niño al centro escolar.

- Motivos de asistencia a la Escuela Infantil.

- Sentido, finalidad e importancia que otorgan los pequeños a la Escuela Infantil.

- La función preparatoria de la Escuela para el futuro ("aprender, trabajar, estudiar").

- La función lúdica ("jugar, pasarlo bien”).

- La creación de lazos de amistad ("estar con mis amigos").

- Grado de satisfacción con la Escuela Infantil.

- Gustos y preferencias en el contexto escolar.

- Actividades vinculadas a contenido lúdico dentro del aula escolar.

- Actividades vinculadas a contenido lúdico fuera del aula escolar.

- Aspectos hacia los que los pequeños muestran desagrado.

$\bigcirc$ La gestión de la convivencia en las aulas.

- El manejo y control conductual.

- Tareas/deberes escolares.

- Juguetes o materiales en mal estado.

- $\quad$ Autonomía y libertad en la escuela.

- Nivel de participación/ implicación de los pequeños en la planificación de la actividad pedagógica.

- Aspectos organizativos.

○ La organización de agrupamientos inter-nivelares.

- Participación de las familias.

○ Deseo o demanda de participación de las familias por parte de los niños.

- Realidad vivenciada por los niños.

- Propuestas de mejora. 


\section{Resultados obtenidos}

Bienestar del niño en el contexto escolar: ¿les gusta asistir a la Escuela Infantil?

Los niños del último curso de Educación Infantil coinciden en señalar que les gusta asistir a la Escuela Infantil, concibiendo la escuela como un lugar donde fraguar relaciones de amistad con iguales, jugar y acercarse a un contenido pre-académico, denominado por ellos "hacer trabajos" y que resulta de su agrado e interés. Así lo ponen de manifiesto unos niños la conversación mantenida con la investigadora:
Entrevistadora: ¿Os gusta venir al cole?
Niños 1 y 2: Sí
Entrevistadora: ¿Por qué os gusta venir al cole?
Niña 1: Porque podemos hacer trabajos, pasar tiempo con nuestros amigos y pasarlo bien.
Niño 2: Porque hay dos amigos que me gustan y siempre jugamos juntos en el patio.

\section{Sentido y finalidad que otorgan los pequeños a la Escuela Infantil}

El discurso de los niños en esta dimensión enfatiza la importancia del aprendizaje de contenido académico, acuñando en su conversación términos específicos en los diálogos que establecen con la investigadora como "aprender, trabajar y estudiar" y que entienden como esenciales para su futuro. Se presenta así una perspectiva donde emergen creencias y preocupaciones de adultos vinculadas con la vida laboral, dejando en un segundo plano la función lúdica. Dicha influencia se evidencia en conversaciones como las que recogemos a continuación y que otorgan a la Escuela Infantil un carácter preparatorio. De esta forma lo expresan los pequeños en dos extractos de conversaciones:

Entrevistadora: ¿Para qué se va a la escuela?

Niño1: Para aprender cosas que no se pueden en casa

Niño 2: Para aprender a trabajar y aprender a leer.

Yo: ¿Creéis que es importante ir a la escuela?

Los dos: Sí

Entrevistadora: ¿Por qué?

Niño 1: Si, para aprender mucho

Niño 2: Porque hay que venir al cole y aprender para cuando seamos mayores. Si no aprendes, de mayores no podemos encontrar nada fijo.

Entrevistadora: ¿Para qué se va a la escuela?

Niño 1: Para cuando sean mayores poder aprender el trabajo que quieran como el de bombero.

Niño 2: Para hacernos niños listos y trabajadores

Entrevistadora: ¿Creéis que es importante ir a la escuela? ¿por qué?

Niño 1: Sí, para saber muchas cosas y después ir al colegio de los mayores. 
Niño 2: Sí, ya lo he dicho antes... Para hacernos listos y trabajadores. Si estudiamos mucho, mucho, mucho luego de mayor pues podemos trabajar $\mathrm{y}$ tener dinero.

Los pequeños a través de la actividad pictórica expresan sus ideas de forma clara e inequívoca incidiendo en aquellos aspectos que les son más significativos y que resultan representados finalmente en el folio. En este sentido, nos parece ilustrativo el dibujo que se recoge a continuación, y que refleja la visión del niño sobre la escuela como un lugar de aprendizaje y transmisora de conocimientos, preparatoria para el futuro:

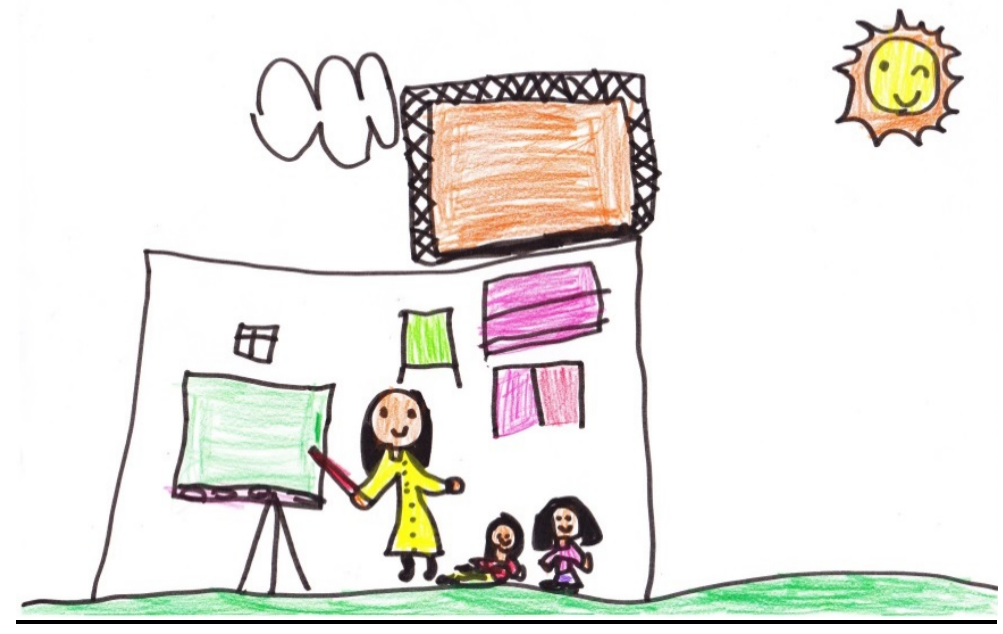

Dibujo I. Sentido y finalidad de la escuela

\section{Grado de satisfacción con su escuela}

Los niños dicen sentirse satisfechos con su escuela, destacando nuevamente la parte lúdica, académica y social que se desprende del colegio. Los pequeños destacan su predilección por el juego en el contexto escolar, incidiendo también en la importancia de poseer unos referentes sociales (adultos y compañeros) seguros, destacando la buena relación que poseían con su maestra y las relaciones de amistad que han conseguido entablar en la escuela. Al respecto, los niños en su discurso conceden importancia al espacio escolar, concretamente, a los espacios de juego con los que dicen sentirse satisfechos y donde experimentan actividades lúdicas placenteras.

Entrevistadora: ¿Os gusta vuestra escuela?

Los dos: Sí

Entrevistadora: ¿Y por qué?

Niña 1: Porque tiene un patio muy grande 
Niña 2: Porque hay cosas divertidas

Entrevistadora: ¿Hay cosas divertidas cómo cuáles?

Niña 2: Como ir al patio, ir de excursión y todo eso

Entrevistadora: ¿Y qué es lo que más os gusta de ella?

Niña 1: Los juegos

Entrevistadora: ¿En dónde?

Niña 1: En clase y en el patio

Entrevistadora: ¿Y qué juegos te gustan?

Niña 1: Hacer carreras, jugar en el ordenador y muchas más.

Niña 2: Jugar con $\mathrm{X}$ a unas cositas como la cocinita.

\section{Gustos y preferencias en el contexto escolar}

Los niños destacan su predilección por aquellas actividades donde el juego esté presente con independencia del espacio dónde se realicen. Por ello, dentro del contexto aula, los pequeños refieren su gusto e interés por actividades que vinculan con contenido lúdico, como la pintura, el rincón de informática y el juego por rincones. Siendo, fuera del aula, el patio de recreo el espacio más valorado, seguido del gimnasio, como espacio donde se realizan actividades psicomotrices con un alto contenido lúdico. Así es como lo expresan los niños en varias conversaciones:

Entrevistadora: ¿Qué espacios de la escuela os gustan más? ¿Por qué?

Niño 1: El de informática, porque en el ordenador hay juegos muy divertidos.

Niña2: Jugar en los rincones

Entrevistadora: ¿Qué espacios de la escuela os gustan más? ¿Por qué?

Niño 1: A mí, el gimnasio, ir y hacer lo de los tiburones ¿te acuerdas de eso? Nos tenían que atrapar y nosotros teníamos que correr desde aquí hasta aquí.

Asimismo, es necesario destacar cómo los niños, representan en sus dibujos el juego, sobre todo, en el patio escolar, siendo el espacio más representado por los pequeños. Evidencia de ello es el dibujo que se presenta: 


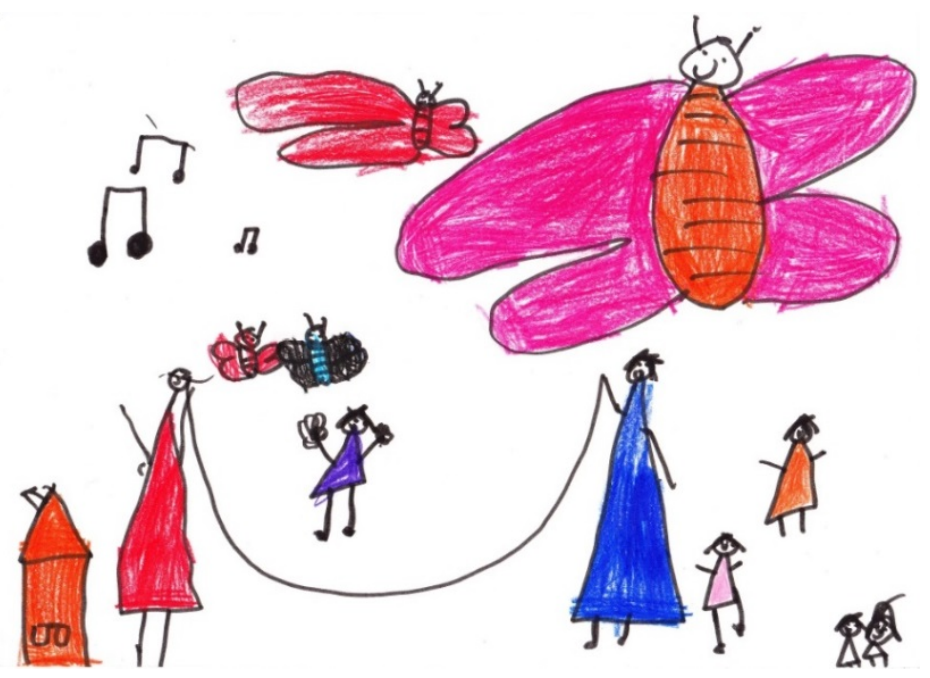

Dibujo II. Gustos y preferencias en el contexto escolar

Aspectos de la escuela hacia los que los pequeños manifiestan desagrado o rechazo

El mantenimiento de la convivencia en las aulas y el manejo conductual se presentan como los contenidos más conflictivos y que generan mayor malestar en los pequeños. Al respecto, los niños señalan su desagrado ante castigos o la aparición de conductas disruptivas en compañeros de clase que interfieren en la dinámica del aula.

Entre los contenidos que señalan les causan malestar o desagrado aparece también la existencia de tareas escolares que les resulta excesivamente complicadas y que limitan la posibilidad de realizar actividades con un componente más lúdico. De esta forma se recoge en la siguiente conversación con niños:

Niña: Lo aburrido para mí es cuando a veces la profe se enfada y nos castiga

Entrevistadora: ¿Y cómo os castiga?

Niño: Pues mira nos sienta en una silla como esa y ya está

Entrevistadora: ¿Y algo más que no te guste?

Niña: La biblioteca

Entrevistadora: ¿por qué?

Niña 2: Porque casi no se leer

Entrevistadora: ¿Y si te leen los cuentos te gusta más?

Niña2: Sí, es muy cansado leer.

Otro aspecto ante el que los niños manifiestan su desagrado es la existencia de juguetes en mal estado o que ya no les son de interés dentro del aula y que se convierte, finalmente, en una demanda explícita de incrementar el componente lúdico en las aulas. Un dibujo que nos parece representativo de lo expresado con anterioridad es el 
siguiente, donde una niña introduce dentro de la clase elementos propios del patio escolar, explicitando de alguna forma sus ganas por incorporar en las dinámicas de aula una mayor presencia del juego.
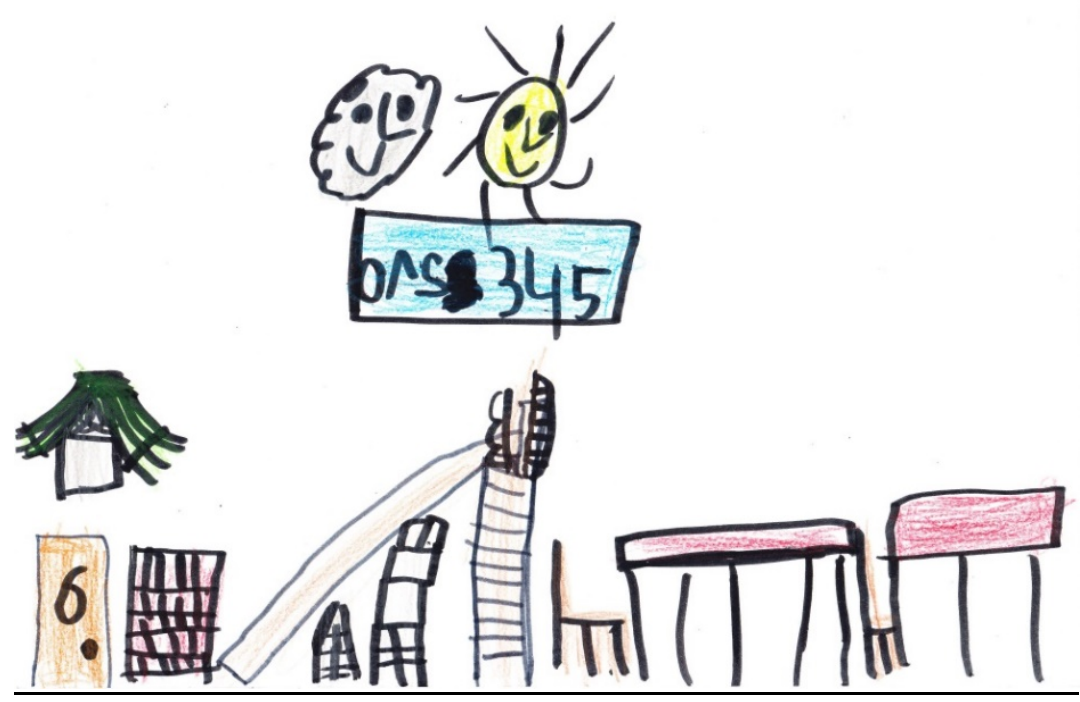

Dibujo III. Deseos de una niña de introducir en el aula el componente lúdico

\section{Autonomía y libertad otorgadas a los niños en la escuela}

Los niños señalan que las dinámicas establecidas dentro del aula han sido instauradas y propuestas por las maestras, sin concederles a los pequeños capacidad de realizar propuestas o elegir una actividad a realizar. En este sentido, nos parece interesante señalar cómo los niños reclaman más capacidad de participación en la elección de temáticas a investigar, conocer o explorar. A continuación se presentan algunas conversaciones representativas que nos muestran el interés de los niños por participar e involucrarse en aquellos asuntos que les afectan:

Entrevistadora: ¿La profesora os deja alguna vez elegir qué actividad o temas queréis trabajar?

Niña 1: No

Niña 2: No

Entrevistadora: ¿Os gustaría?

Niña 1: Sí, yo animales quiero aprender.

Niña 2: Sí, animales también.

Entrevistadora: ¿La profesora os deja alguna vez elegir qué actividad o temas queréis trabajar?

Niño 1: Lo elige ella 
Niño 2: Lo elige ella siempre

Entrevistadora: ¿Os gustaría?

Niño 1: Bueno alguna vez elige entre dos cosas y vota.

Entrevistadora: ¿Y eso os gusta más o preferís que elija ella siempre?

Niño 1: Sí, más

Niño 2: Que podamos elegir

\section{Aspectos organizativos}

La organización de los agrupamientos y el desarrollo de actividades conjuntas con otros grupos de alumnos es un tema que resulta de interés para los pequeños. En este aspecto, es significativo el interés que los niños presentan por realizar actividades conjuntas con niños de otros grupos de diferentes edades, poniendo de manifiesto que suelen participar en actividades comunes con los alumnos del otro grupo de cinco años. Es la posibilidad de realizar actividades conjuntas con alumnado de Primaria las que les resultan más atractivas, ya que dicen identificarse más con los mayores a quienes consideran más sabios pudiéndose acercar así a conocimientos y experiencias que podrían facilitar una adecuada transición escolar a la etapa de Educación Primaria. Así se pone de manifiesto en las siguientes conversaciones:

Entrevistadora: ¿A veces os juntáis con niños de otras clases para hacer actividades o trabajos de clase?

Niño 1 y 2: No

Entrevistadora: ¿Os gustaría juntaros con niños más mayores o más pequeños?

Niño 1: $\mathrm{Si}$, los mayores porque ya son mayores como nosotros

Niño 2: Sí, los niños de la clase de Primaria. Los pequeños lo destrozan todo y los mayores saben más

\section{Participación de las familias en el contexto escolar}

La participación de las familias en el contexto escolar es un aspecto altamente deseado por todos los niños entrevistados. Sin embargo, los pequeños señalan que la presencia de familiares en el centro se limita a eventos excepcionales y las entradas y salidas del centro, entendiendo su participación dentro de las aulas como algo deseable pero difícil de realizar, dada la normativa del centro escolar. Una conversación donde se pone de manifiesto esta situación es la siguiente, donde se refleja la capacidad de adaptación de los pequeños y cómo finalmente, sus deseos quedan supeditados a una normativa escolar impuesta por el adulto.

Entrevistadora: ¿Vuestra mamá o vuestro papá entran alguna vez al aula para ver como trabajáis o hacer actividades con vosotros?

Niño 1: No

Niño 2: No, aunque una vez vinieron pero mis abuelitos para venir a recogerme. 
Entrevistadora: ¿Os gustaría?

Los dos: Sí

Niño 2: A mi sí me gustaría pero como no nos dejan a mí me da igual ahora.

\section{Propuestas de mejora para la escuela}

Para finalizar las conversaciones mantenidas con los niños se les solicitó que realizasen propuestas de mejora para la Escuela Infantil. Sus sugerencias giraron en torno al incremento de espacios de juego tanto dentro como fuera del aula, la mejora y reposición de juguetes estropeados y el incremento de su capacidad de acción e intervención en el espacio escolar, demandando hacer una escuela donde sus obras pudiesen decorar pasillos y aulas, una demanda que recoge la necesidad también de hacer suyo, de personalizar un espacio del que debieran ser protagonistas. En esta línea proponen también la presencia de mascotas en la clase siguiendo la propuesta de personalizar un espacio en el que pasan tanto tiempo. Constancia de estas propuestas son el extracto de conversación y del dibujo que a continuación se presentan:

Entrevistadora: ¿Qué cambiaríais de la escuela?

Niño 1: Yo la decoraría muy bien, ponerla purpurina, poner dibujos

Entrevistadora: ¿Y tú?

Niña/o 1: Yo pondría marionetas, un tobogán y una casita para jugar.

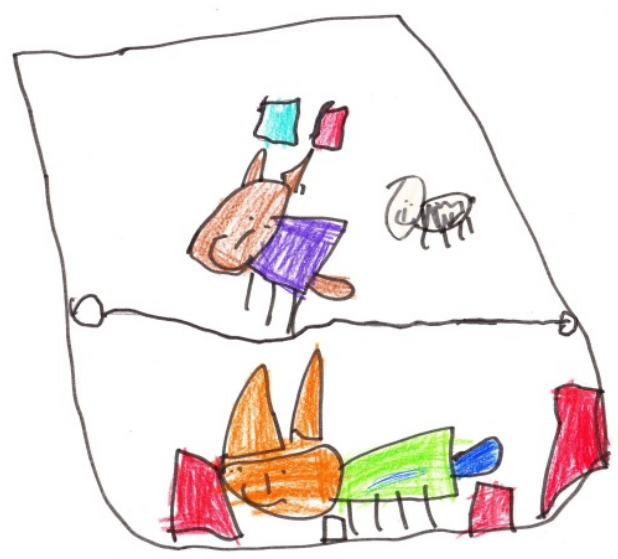

Dibujo IV. Propuestas de mejora para la Escuela Infantil

\section{Conclusiones}

Recoger la visión de los niños sobre aquellos temas que les afectan y de los que son protagonistas, supone reconocer su capacidad de acción e intervención en aquellos contextos donde se desenvuelven a diario y del que son expertos (Harcourt y 
Einarsdottir, 2011). La Escuela Infantil se presenta como un contexto donde los pequeños pasan largo tiempo, experimentan, descubren y comparten vivencias y situaciones ricas, convirtiéndose en los principales actores de un escenario en el que pocas veces se escucha su voz. No tener en cuenta sus aportaciones en la investigación educativa supone prolongar la existencia de sesgos en torno al conocimiento de la realidad educativa que experimentan los niños, cuyo estudio ha tenido más presente la perspectiva adulta que la infantil (Christensen y Prout, 2002).

La investigación que se ha presentado refuerza la importancia y necesidad de recoger la opinión de los niños desde las edades más tempranas para poder instaurar procesos de innovación y mejora escolar que tomen en consideración las aportaciones infantiles en aquellas cuestiones que les afectan, en un intento de que su voz tenga poder (Dahlberg, Moss y Pence, 1999) y pueda tener finalmente un impacto práctico, real y formal en el centro escolar.

Particularmente, el estudio a pesar de las limitaciones derivadas de una muestra reducida, nos ha permitido comprobar que los niños tienen la capacidad de hacer partícipe al adulto de sus gustos, preferencias, deseos, demandas y propuestas de mejora de la Escuela Infantil con claridad y determinación siempre y cuando el adulto sea capaz de seleccionar técnicas de escucha adecuadas que superen las barreras o limitaciones expresivas propias de la edad. Al respecto, las técnicas del dibujo y las conversaciones con niños utilizadas han facilitado el desarrollo de un proceso de escucha efectivo a la infancia en esta investigación..

Asimismo, los resultados ponen de manifiesto la demanda de los niños que asisten al centro escolar donde se ha desarrollado el estudio, de una Escuela Infantil diferente a la que conocen. Si bien dicen sentirse a gusto en la Escuela, de su discurso se infiere la importancia que conceden al juego y al espacio escolar, solicitando una mayor presencia del componente lúdico dentro y fuera de las aulas escolares. En el análisis efectuado, se evidencia la existencia de un pensamiento divergente en los niños participantes respecto del pensamiento del adulto en torno a las siguientes cuestiones: los aspectos organizativos que rigen la dinámica escolar, señalando su deseo de contar con la presencia y la participación de las familias en la aula; la reivindicación de actividades o proyectos que supongan el establecimiento de agrupamientos inter niveles o entre etapas educativas que aproximen a los niños al contacto y conocimiento de otros niños de mayor edad, y que entienden como fuente de sabiduría, así como la demanda explícita de incrementar su autonomía y capacidad de participación en la elección de temáticas a investigar, conocer o explorar en la aula. Finalmente, plantean la necesidad de personalizar un contexto, como es el escolar, en el que pasan tanto tiempo, pudiéndose incrementar así el sentido de pertenencia infantil a un ámbito donde la presencia del adulto y su pensamiento sigue dominando las prácticas pedagógicas. 


\section{Referencias bibliográficas}

ALDERSON, P. (2000). Young Children's Rights: Exploring Beliefs, Principles and Practice. London: Jessica Kingsley Publishers.

APUD, A. (2007). Cuaderno de formación del profesorado sobre participación infantil. Enrédate. UNICEF. [formato html]. http://www.enredate.org/docs/ doc4abcaf8c94cf65.46858126.pdf (Consultado el 5 de Septiembre de 2010).

ARGOS, J. EZQUERRA, P Y CASTRO, A. (2011). Escuchando la voz de la infancia en los procesos de cambio e investigación educativos. Aproximación al estudio de las transiciones educativas entre las etapas de Educación Infantil y Educación Primaria, Revista Iberoamericana de Educación, 54(5), [formato html]. http://www.rieoei.org/deloslectores/3988Argos.pdf (Consultado el 15 de Febrero de de 2012).

BERTOLINI, M. (2011). Hacia otros modos de pensar la educación infantil: La posibilidad de una (otra) pedagogía de la escucha, de la espera, del quizás... Comunicación del XII Congreso Internacional de Teoría de la Educación, Barcelona. [formato html]. http://www.cite2011.com/Comunicaciones/ Escuela/119.pdf (Consultado el 25 de Octubre de 2013).

BORZONE, A. M., Y ROSEMBERG, C. R. (2000). ¿Qué aprenden los niños cuando aprenden a hablar?. El desarrollo lingüístico y cognitivo en los primeros años. Buenos Aires: Aique.

BRUNER, J (1996). The culture of education. Cambridge, MA: Harvard University Press.

CABALlERO, M.A; CABELlO, P; DE DIEGO, E; HERNÁNDEZ-PIZARRO, M.L; PARRA, J.M Y ORTIZ, P. (1999). El practicum de Pedagogía: evaluación crítica de la opinión del alumnado, Revista Electrónica Interuniversitaria de Formación del Profesorado 2(1) [formato html]. http://www.uva.es/aufop/publica/revelfop/ 99-v2n1.htm (Consultado el 13 de Octubre de 2014).

CASTRO, A. EZQUERRA, P. Y ARGOS, J. (2012a). La mirada infantil sobre la transición educativa. Cuadernos de Pedagogía, 419, 28-31.

CASTRO, A. EZQUERRA, P. Y ARGOS, J. (2012b). La transición entre la Escuela de Educación Infantil y la de Educación Primaria: perspectivas de niños, familias y profesorado. Revista Española de Pedagogía, 253, 537-552.

CLARK, A. Y MOSS, P.(2001). Listening to Young Children: The Mosaic Approach. London: National Children's Bureau.

CLARK, A, MCQUAIL, S Y MOSS, P (2003). Exploring the Field of Listening to and Involving Young Children. Research Report 445. DfES

CHRISTENSEN, P. Y PROUT, A. (2002). Working with Ethical Symmetry in Social Research with Children. Childhood, 9 (4), 477-497. 
COTERÓN, J., FRANCO, E., Y GIL, J. (2012). Opinión del alumnado sobre la implantación del Espacio Europeo de Educación Superior en estudios de Ciencias de la Actividad Física y del Deporte. Revista Complutense de Educación, 23(1), 191-206.

DALHBERG, G; MOSS, P. Y PENCE, A. (1999). Beyond Quality in Early Childhood Education and Care: Postmodern Perspectives.London and Philadelphia, PA: Falmer Press.

DOCKETT, S Y PERRY,B. (2005). Starting school in Australia is a "bit safer, a lot easier and more relaxing": Issues for parents from culturally and linguistically diverse backgrounds, Early Years, 25(3), 271 - 281.

DOVAL, M.I.; MARTINEZ-FIGUEIRA, M.E. Y RAPOSO, M. (2013). La voz de sus ojos: la participación de los escolares mediante Fotovoz. Revista de Investigación en Educación, 11(3), 150-171.

FIELDING, M. (2011). La voz del alumnado y la inclusión educativa: una aproximación democrática radical para el aprendizaje intergeneracional. Revista Interuniversitaria de Formación del Profesorado, 70 (25,1), 31-61.

FINE, M. (1991) Framing Dropouts: Notes on the Politics of an Urban Public High School. Albany: Suny Press.

GUZMAN, M. (2013). La oreja que escucha a las niñas y los niños. Revista Infancia: Educar de 0 a 6 años, 138, 34-38.

HARCOURT, D. Y EINARSDÓTTIR, J. (2011). Introducing children's perspectives and participation in research, European Early Childhood Education Research Journal, 19(3), 301-307.

HART, R. (1993). La participación de los niños: de la participación simbólica a la participación auténtica. Editorial Nueva Gente, Bogotá, Colombia.

JIMÉNEZ, M.A. Y NAVARIDAS, F. (2012). Cómo son y qué hacen los "maestros excelentes": la opinión de los estudiantes. Revista Complutense de Educación, vol. 23(2), 483-485.

LIEBEL, M, Y SAADI, I.(2012). La participación infantil ante el desafío de la diversidad cultural. Desacatos, 39, 123-140. [formato html]. http://www.scielo.org.mx/scielo.php?script=sci_arttext\&pid=S1405-

92742012000200009\&lng=es\&tlng=es (Consultado el 11 de Octubre de 2014).

MESSIOU, K. (2013). El compromiso con la voz del alumnado: uso de un marco de trabajo para abordar la marginación en las escuelas. Revista de Investigación en Educación, 11(3), 97-108.

MILLER, J.(1997). Never too young: how young children can take responsibility and make decisions. London: National Early Years Network/Save the Children.

MITRA, D.L. (2003). Student voice in school reform: reframing student-teacher relationships, McGill Journal of Education, 38(2), 289-304. 
MOLERO, D. (2007). Rendimiento académico y opinión sobre la docencia del alumnado participante en experiencias piloto de implantación del Espacio Europeo de Educación Superior. Revista Electrónica de Investigación y Evaluación Educativa, 13(2), 175-190.

NOVELLA, A.M. (2012). La participación infantil: Concepto dimensional en pro de la autonomía ciudadana. Revista Teoría de la Educación: Educación y Cultura en la Sociedad de la Información, 13(2), 380-403.

RUIZ DE MIGUEL, C. Y OLIVEROS MARTÍN-VARÉS, L. (2006). La opinión del alumno de la facultad de educación acerca de la metodología docente. Revista Complutense de Educación, 17(1),29-48. [formato html]. http://www.ucm.es/ BUCM/revistas/edu/11302496/articulos/RCED0606120029A.PDF(Consultado el 11 de Octubre de 2014)

ORGANIZACIÓN DE LAS NACIONES UNIDAS (ONU) (1989).Convención de los Derechos del Niño. [formato html]. http://www.un.org/es/events/childrenday/ pdf/derechos.pdf (Consultado el 9 de Octubre de 2014)

ORGANIZACIÓN DE LAS NACIONES UNIDAS (ONU) (2005), Convención de los Derechos del Niño. Observación General N ${ }^{\circ}$ 7: Realización de los derechos del niño en la primera Infancia, [formato html]. http://www2.ohchr.org/ english/bodies/crc/docs/AdvanceVersions/GeneralComment7Rev1_sp.pdf (Consultado el 11 de Octubre de 2014).

ORGANIZACIÓN DE LAS NACIONES UNIDAS (ONU) (2009), Convención de los Derechos del Niño. Observación General $N^{\circ}$ 12: El derecho del niño a ser escuchado, [formato html]. http://reddedalo.files.wordpress.com/2012/12/ observacic3b3n-general-12-cdn.pdf (Consultado el 10 de Octubre de 2014).

PARRILLA, A. Y DE LA FUENTE, A. (2013). Foto voz: La escuela vista por los pequeños. Revista Infancia: Educar de 0 a 6 años, 138, 22-23.

PASCAL, C. Y BERTRAM, T.(2009). Listening to young citizens: the struggle to make real a participatory paradigm in research with young children, European Early Childhood Education Research Journal, 17,(2), 249-262.

RINALDI, C. (2001). A pedagogy of listening: a perspective of listening from Reggio Emilia. Children in Europe, 1, 2-5.

RUBIANO, E. (2010). A la escucha de la infancia, Educere, 14(49), 297-303.

RUDDUCK, J. Y FLUTTER, J. (2000). Pupil participation and pupil perspective: 'carving a new order of experience', Cambridge Journal of Education, 30(1), 7589.

RUDDUCK, J. Y FLUTTER, J. (2007). Cómo mejorar tu centro escolar dando la voz al alumnado. Madrid: Morata.

SÁEZ, F. J. (2000). La opinión de los estudiantes universitarios sobre el método docente de las facultades de ciencias. Revista de Investigación Educativa, 18,(1), $37-43$. 
TRILLA, J. Y NOVELLA, A. (2011). Educación y participación social de la infancia. Revista Iberoamericana de educación, 26, 137-166.

YOUNG, L. Y BARRETT, H. (2001). Issues of access and identity: adapting research methods with Kampala street children, International Journal of Childhood, 8 (3), 383-395.

\section{Correspondencia con los autores}

Ana CASTRO ZUBIZARRETA

Universidad de Cantabria

Facultad de Educación

Avda. Los Castros s/n

39005 Santander

e-mail: castroza@unican.es

Nuria del Mar MANZANARES CEBALLOS

Universidad de Cantabria

Facultad de Educación

Avda. Los Castros s/n

39005 Santander

e-mail: nuria-del-mar.manzanares@alumnos.unican.es 